\title{
Fiber-Wireless-Fiber Terminals for Optical Wireless Communication over Multiple Bands
}

\author{
Ravinder Singh, Andy Schreier, Grahame Faulkner and Dominic O’Brien \\ Department of Engineering Science, University of Oxford, OX1 3PJ, UK. \\ Email: ravinder.singh@eng.ox.ac.uk (ORCID: 0000-0002-2646-4204)
}

\begin{abstract}
This paper studies the multi-wavelength capability of our recently developed tracking and beam-steering fiber-wirelessfiber (FWF) terminals for simultaneous, full-duplex optical wireless communication over telecommunication bands widely used in optical access networks. Tracked terminal-to-terminal concurrent data transmission over $\mathrm{O}$ and $\mathrm{C}$ bands is demonstrated.
\end{abstract}

Keywords-Optical Wireless Communication, Localization and Tracking, Fiber-Wireless-Fiber, Beam-steering, Passive Optical Networks

\section{INTRODUCTION}

As $5 \mathrm{G}$ communication systems are rolled out attention is moving towards "Beyond 5G" technologies. Critical to this endeavour will be accessing new regions of spectrum, and technologies that can use these regions to provide ultra-high data rate wireless communications for demanding applications such as virtual and augmented reality. Fiber optic communication technologies with relatively mature modulation, detection and multiplexing techniques have demonstrated the capability of Tbit/s in point to multipoint architectures [1]. Further development of ultra wideband optical wireless communication (OWC) technology, in a specific form of $\mathrm{FWF}$, is an area of particular interest. In FWF, the light from the fiber is collimated, forming a narrow parallel beam which is steered from a transmitter (Terminal-A) to a receiver (Terminal-B as shown in Fig. 1(a)), where additional beam steering guides the light beam back into a fiber for detection through fiber optic transceivers. The beam steering relies on a high precision tracking system.

Recent research into OWC technology has relied on the use of fast steering mirrors (FSMs) [2][3], spatial light modulators (SLMs) [4] and passive diffraction gratings [5]. Ultra-high data rates have been demonstrated with limited coverage using FWF technology. However, spectral capability has received little attention, which is important for compatibility with wide range of optical bands used in access networks. Transmitting multiple wavelengths simultaneously provides the opportunity to design a system architecture with more degrees of freedom [6]. Multiple wavelengths can be used to (i) increase the transmission rate, (ii) provide multi-user access, and (iii) distribute different services and their corresponding classes within the same access network.

Recently, we have presented a compact FWF terminal architecture shown in Fig. 1(b), which uses FSMs and low-cost cameras to provide combined functionality of beam steering and localization [7]. A fully assembled terminal is shown in Fig. 1(c). This has been used to transmit $>1 \mathrm{Tbit} / \mathrm{s}$ in the $\mathrm{C}$ band, with automated tracking between terminals [8].
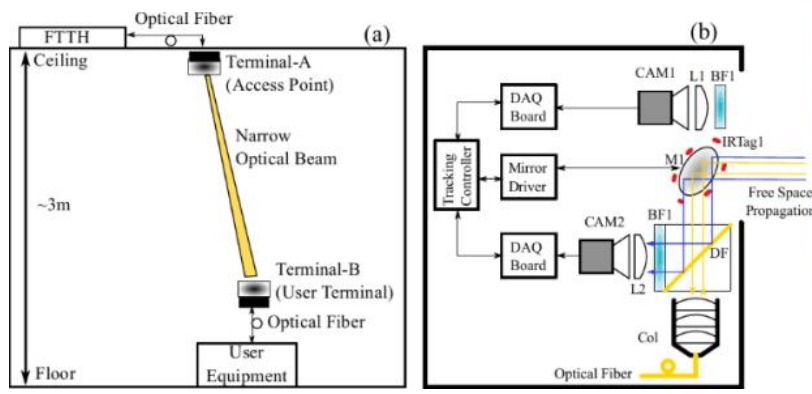

Fig. 1: (a) An indoor FWF setup, (b) terminal architecture, and (c) a fully assembled terminal.

This paper studies operation over two widely space wavelengths simultaneously. Full-duplex 20.6 Gbit/s down-link and 13.425 Gbit/s up-link data transmissions are demonstrated by simultaneously using standard SFP+ transceivers operating in the $\mathrm{O}$ and $\mathrm{C}$ bands. The results show that a pair of terminals could provide full data rates over a coverage of $4.9 \mathrm{~m} 2$. The additional losses observed in the $\mathrm{O}$ band were minimal, even though the tracking system was calibrated for $1550 \mathrm{~nm}$ operation.

\section{MEASUREMENTS}

\section{A. Experimental Setup}

In order to test the terminal operation at 1310nm and simultaneous full-duplex operation over $\mathrm{O}$ and $\mathrm{C}$ bands, the experimental setup shown in Fig. 2 was used. The 1310nm down-link (DL) and up-link (UL) were driven by identical SFP+ transceiver modules (FS SFP-10G-ER40) plugged into two separate bit error ratio testers (BERT), Spectronix Eye-BERT and Agilent N5980A, respectively. The $1310 \mathrm{~nm}$ DL was set to $10.3 \mathrm{Gbit} / \mathrm{s}$ and the $1310 \mathrm{~nm} \mathrm{UL}$ was set to $3.25 \mathrm{Gbit} / \mathrm{s}$. The rates were limited by the capability of the BERTs used. The DL and UL at 1310nm used 231 bit long pseudo random bit sequences (PRBS). The 1550nm link was driven by another SFP+ module (Finisar FTLX1871M3BCL) plugged into an evaluation board (FDB-1032-SFP+) which was connected to an arbitrary waveform generator (AWG700002A) and an oscilloscope (KeySight MSOV334A). A 215 bit long PRBS signal was used for the $1550 \mathrm{~nm}$ DL and UL which were set to $10.3 \mathrm{Gbit} / \mathrm{s}$. Due to the limited number of sources and detectors, the $1550 \mathrm{~nm}$ DL was optically amplified at terminal-B and transmitted back to terminal-A to create an UL and full-duplex transmission. 
The full duplex operation was enabled by using pairs of circulators (6015-3-APC, CIR1310-APC), while the $1310 \mathrm{~nm}$ and $1550 \mathrm{~nm}$ links were multiplexed using a wavelength division multiplexer (WD1350A) at each terminal. This resulted in an aggregate DL data-rate of $20.6 \mathrm{Gbit} / \mathrm{s}$ and an aggregate UL data-rate of 13.425 Gbit/s. At DL and UL transmitters, approximately $2 \mathrm{dBm}$ optical power was launched into the single-mode fibers.

\section{B. Results and Discussions}

The receiver sensitivities for the full-duplex data transmissions were evaluated in a received power

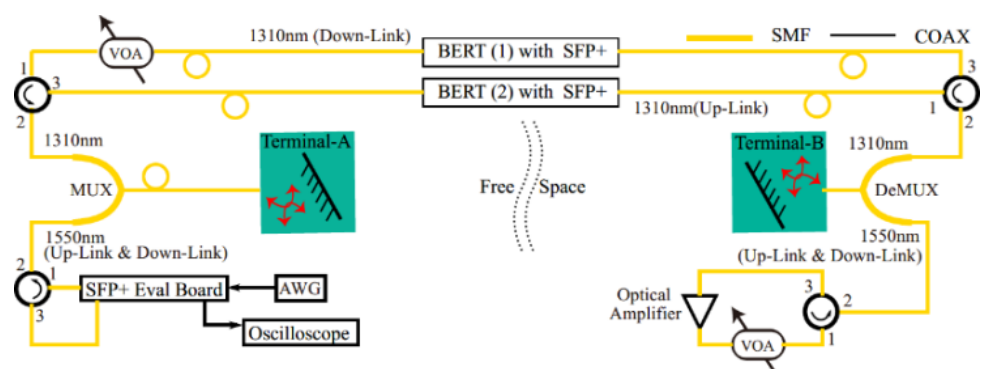

Fig. 2: Experimental setup for testing spectral agility of the FWF terminals. versus bit error ratio (BER) measurement, with terminals separated by approximately $4 \mathrm{~m}$ distance, facing each other. The transmit power was stepwise reduced by variable optical attenuators (VOA50-APC) and the BER was measured for the 1310nm DL and UL, as well as the combined DL+UL 1550nm transmissions. The results are shown in Fig. 3(a). The solid and dashed lines show the BER for simultaneous and independent data transmissions, respectively. The results in Fig. 3(a) indicate that the 1310nm DL and UL, which uses SFP-10G-ER40 SFP+ module, requires approximately $-21.5 \mathrm{dBm}$ and $-22.5 \mathrm{dBm}$ received powers for a BER of 1x10-3, which is a widely used reference BER required at the input of super-FEC decoders recommended in ITU-T G.975.1. This $1 \mathrm{~dB}$
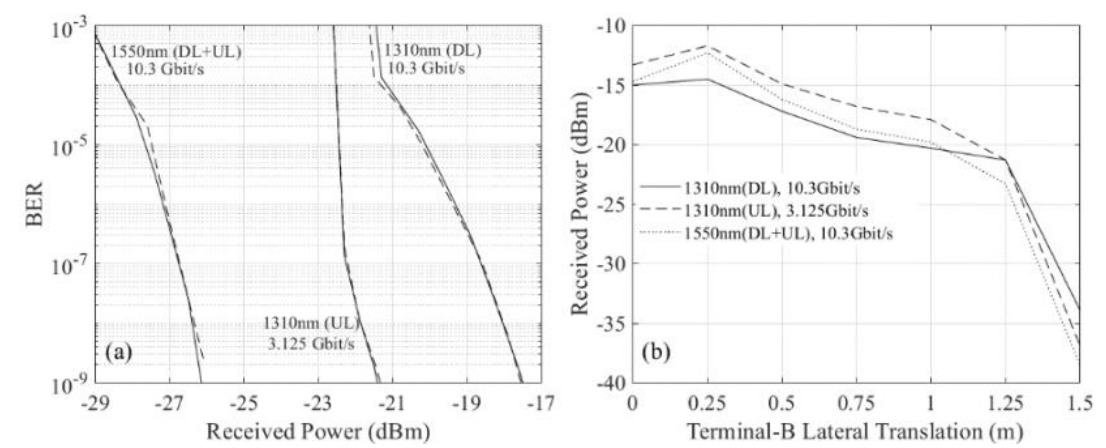

Fig. 3: (a) BER vs received power ( $\mathrm{dBm}$ ) for sensitivity evaluation. The solid and dashes lines show the BER in simultaneous and independent data transmission modes, respectively. (b) The received power for each link at discrete Terminal-B locations. difference arises due to lower data-rate used on the $1310 \mathrm{~nm}$ UL. The $1550 \mathrm{~nm}$ combined DL+UL, which uses the FTLX1871M3BCL $\mathrm{SFP}+$ module, requires approximately $-29 \mathrm{dBm}$ received power for a BER of $1 \times 10-3$. This required power difference in the $1550 \mathrm{~nm}$ and $1310 \mathrm{~nm}$ links arise due to higher sensitivity receiver used in the FTLX1871M3BCL module, which provides a larger link budget. These results show that simultaneous data transmission does not affect the BER performance.

In order to study the achievable coverage in an indoor environment, Terminal-B was translated from 0 to $1.5 \mathrm{~m}$ in a lateral direction w.r.t. Terminal-A with automated tracking enabled and the received power was measured for each position of the Terminal-B. The measured received power levels for each of the link are shown in Fig. 3(b). These indicate that the received power levels are sufficient to maintain a BER $\leq 1 \times 10-3$ for a lateral translation of Terminal-B up to $1.25 \mathrm{~m}$. This results in an approximate cell size of up to $4.9 \mathrm{~m} 2$ for a terminal-to-terminal full-duplex simultaneous data transmission over $\mathrm{O}$ and $\mathrm{C}$ bands.

\section{CONCLUSIONS}

The results indicate that FWF architecture has the potential to provide wireless connection for multiple services across the optical spectrum in access networks. A broader range of operation might be achieved by using reflective collimators instead of lens-based. However, this work shows that only modest penalties are incurred for operation across a very broad range of wavelengths.

\section{REFERENCES}

[1] Peter J. Winzer, David T. Neilson, and Andrew R. Chraplyvy, "Fiber-optic transmission and networking: the previous 20 and the next 20 years [Invited]," Opt. Express 26, 24190-24239, 2018.

[2] Paul Brandl, Alexander Weiss, and Horst Zimmermann, "Automated alignment system for optical wireless communication systems using image recognition," Opt. Lett. 39, 4045-4048, 2014.

[3] K. Wang, A. Nirmalathas, C. Lim and E. Skafidas, "4x12.5 Gb/s WDM Optical Wireless Communication System for Indoor Applications," in Journal of Lightwave Technology, vol. 29, no. 13, pp. 1988-1996, July1, 2011.

[4] A. Gomez et al., "Design and Demonstration of a $400 \mathrm{~Gb} / \mathrm{s}$ Indoor Optical Wireless Communications Link," in Journal of Lightwave Technology, vol. 34, no. 22, pp. 5332-5339, 15 Nov.15, 2016.

[5] T. Koonen, F. Gomez-Agis, F. Huijskens, K. A. Mekonnen, Z. Cao and E. Tangdiongga, "High-Capacity Optical Wireless Communication Using TwoDimensional IR Beam Steering," in Journal of Lightwave Technology, vol. 36, no. 19, pp. 4486-4493, Oct.1, 2018.

[6] F. Feng, P. Sangwongngam, G. Faulkner, and D. O'Brien, "Wide fieldof-view optical broadcasting for bi-directional indoor optical wireless communications employing pam-4 modulation," Optics Letters, vol. 44,no. 24, pp. 6009-6012, 2019.

[7] R. Singh et al, "Desing and Characterisation of Terabit/s Capable Compact Localisation and Beam-steering Terminals for Fiber-Wireless-Fiber Links", submitted to Journal of Lightwave Technology, March 2020.

[8] Y. Hong et al, "Beyond Terabit/s WDM Optical Wireless Transmission using Wavelength-Transparent Beam Tracking and Steering ", to appear in OSA Conference on Optical Fiber Communication (OFC), 2020. 